\title{
Viscosupplementation for knee osteoarthritis: a focus on Hylan G-F 20
}

This article was published in the following Dove Press journal: Orthopedic Research and Reviews

\author{
David Webb' \\ Poobalan Naidoo 2,3 \\ 'Pattacus Medical, Randburg, South \\ Africa; ${ }^{2}$ Medical Affairs, Sanofi House, \\ Midrand, South Africa; ${ }^{3}$ Department of \\ Health Informatics, School of Health \\ Professions, Rutgers, State University \\ of New Jersey, NJ, USA
}

\begin{abstract}
Knee osteoarthritis is a chronic degenerative joint disease characterized by destruction of articular cartilage with resultant para-articular bone changes. It is a major cause of disability in older persons and is managed by surgical and nonsurgical interventions. Pharmacotherapy includes acetaminophen, nonsteroidal anti-inflammatory agents, and intra-articular steroids. Another treatment option is viscosupplementation with intra-articular injection of hyaluronan (HA). The full mechanism of action of exogenous HA is uncertain, but studies indicate that it may promote endogenous HA production, reduce inflammation, prevent degeneration of cartilage and promote cartilage regeneration. Clinically, HA may improve symptoms of osteoarthritis and delay time to total knee replacement surgery. However, clinical studies are heterogenous and of varying quality, and thus there is a need for more robust studies to determine the place of viscosupplementation in the management of knee osteoarthritis.
\end{abstract}

Keywords: knee osteoarthritis, viscosupplements, hylan, Hylan G-F 20

\section{Introduction}

Knee osteoarthritis (OA), is a chronic degenerative joint disease, characterized clinically by gradual development of fluctuating joint pain, swelling, stiffness, and loss of motion. ${ }^{1}$ Prevalence increases with age and OA is a leading cause of disability. Worldwide, the prevalence among individuals aged 60 years and older is estimated at $18 \%$ of women and $10 \%$ of men. ${ }^{2,3}$ Approximately $80 \%$ of these individuals will have some degree of limitation of movement, and 1 in 4 will be restricted in major activities of daily living. ${ }^{2}$

Surgical and nonsurgical therapies are available for the management of OA. Nonsurgical interventions include patient education, physical therapy, weight loss, and low-impact exercises. However, many patients will require pharmacotherapy for pain and limitation of movement. Commonly used options include paracetamol, nonsteroidal anti-inflammatory drugs (NSAIDs), and intra-articular injection of corticosteroids. Opioids and nonnarcotic analgesics may be prescribed for those with refractory pain. ${ }^{4}$ However; all of these pharmacotherapies are associated with the potential for adverse effects, especially with long-term use.

Viscosupplementation with intra-articular hyaluronans (HAs) is the latest pharmacological treatment for OA. This paper will explore the science and clinical utility of viscosupplementation for knee OA.
Correspondence: Poobalan Naidoo Medical Affairs, Sanofi House, 44 on Grand Central Office Park, 2 Bond Street, Grand Central Ext. I, Midrand 1685, South Africa

Email poobalan.naidoo@sanofi.com 


\section{Pathological mechanisms in OA}

The pathogenesis of knee OA is characterized by an imbalance between synthesis and degradation of cartilage matrix, resulting in a slow degradation of cartilage occurring over several years. Loss of articular cartilage is accompanied by subchondral bone remodeling, osteophyte formation, and inflammation of the synovial membrane. There is increasing evidence that subchondral bone plays an important role in the pathogenesis of OA. Changes in subchondral bone, as evidenced by MRI, are detected early in the course of OA and may be an appropriate target for future OA therapies. ${ }^{5}$

Various factors produced by the synovium and chondrocytes, which include cytokines, growth factors, aggrecanases, and matrix metalloproteinases (MMPs), play an important role in regulation of this balance between synthesis and degradation. ${ }^{1}$

The extracellular matrix of articular cartilage consists of water and a collagenous framework containing complexes of glycosaminoglycans, proteoglycans (PG), and hyaluronic acid (HA). One important PG is aggrecan (cartilage-specific proteoglycan core protein or chondroitin sulfate proteoglycan 1), which binds HA and provides compressibility and elasticity to cartilage. One of the first pathological changes in knee OA is degradation of aggrecan by aggrecanases, causing cartilage erosion. In conjunction with increased collagen turnover rate, changes in the extracellular matrix include reduced content and altered composition of PG, increased water content and reduced size, and decreased concentration of HA molecules. ${ }^{1}$

Cartilage destruction in knee OA is potentiated by overexpression of cytokines from the synovium and chondrocytes. These include interleukin (IL)- 1 and tumor necrosis factor- $\alpha$, which play a role in destruction of cartilage and stimulate both their own production and that of other proinflammatory cytokines, such as IL-8, IL-6, IL-11, IL-17, and leukotriene inhibitory factor. Increased production of proteases, prostaglandins, and leukotrienes and RANTES also play a role in inflammation and cartilage destruction.

Increased activation of MMPs and decreased activity of their inhibitors contributes to breakdown of the extracellular matrix. ${ }^{1}$

Proinflammatory cytokines also stimulate the production of nitric oxide (NO), which inhibits the synthesis of collagen and PG, enhances MMP activity, and induces and enhances the susceptibility of the chondrocytes to apoptosis. In an attempt to reverse destruction of the extracellular matrix, synthesis of matrix components, including PG, is increased in the middle and deeper layers of the cartilage. However, because chondrocytes are the only source of matrix components and dead cells are not effectively removed from cartilage, apoptosis significantly depletes matrix production. ${ }^{1}$

Anti-inflammatory cytokines that are found in the synovial fluid of knee OA joints include IL-4, IL-10, and IL-13. Other regulatory proteins that stimulate matrix production in OA include insulin-like growth factor-1, transforming and fibroblastic growth factors, and bone morphogenetic proteins. ${ }^{1}$

In synovial joints, although hyaline cartilage does not have nerve endings, the capsule, ligaments, synovial membrane and subchondral bone are innervated by sensory nerves that are sensitive to both noxious and nonnoxious stimuli. Inflammatory mediators act directly on the nerve endings and reduce their threshold to pain recognition. In knee OA, acute pain arises from activation of the peripheral nerve receptors, whereas chronic pain is associated with ongoing inflammation in peripheral tissues. Significant and sustained chronic pain results in central changes, predominantly in spinal neurons, leading to central sensitization and chronic neuropathic pain. ${ }^{4}$

\section{Synovial fluid and HA in healthy and osteoarthritic joints}

Synovial fluid is essential for healthy joint function. The primary chemical component is HA, a polysaccharide chain with a molecular weight (MW) of 4-10 million Da. HA is composed of repeating units of $\mathrm{N}$-acetylglucosamine and glucuronic acid, and is produced by synoviocytes, fibroblastlike synoviocytes (located within the synovium) and chondrocytes. A healthy knee contains $\sim 2 \mathrm{~mL}$ of synovial fluid with a concentration of HA of $2.5-4.0 \mathrm{mg} / \mathrm{mL} .{ }^{6,7}$

During movement, the fluid acts as a lubricant and shock absorber, protecting the articular cartilage and joint structures from compressive and shear forces and thereby reducing articular cartilage wear. It supplies oxygen and nutrients to the surrounding tissues and removes carbon dioxide and metabolic wastes. The HA molecules act as a filter by restricting the entrance of large plasma proteins into the synovial fluid, while facilitating the passage of small molecules into the joint for maintenance of nutrition. ${ }^{4,6}$

The viscoelastic properties of HA are directly related to its MW and concentration and are shear-dependent. With slow movement and low shear, the linear chains of HA align slowly in the direction of the flow, and HA becomes more viscous, acting as a lubricant. In contrast, with faster impact, such as running or jumping, HA has inadequate time to re-align and has greater shock-absorbing properties. ${ }^{8}$ 
HA also exhibits various anti-inflammatory effects. It inhibits phagocytosis and leukocyte adherence, reduces inflammatory mediators, and reduces release of arachidonic acid from synovial fibroblasts. ${ }^{7}$

In OA, as a consequence of dilutional effects, reduced synthesis and degradation by proinflammatory cytokines, free radicals, and proteinases, the concentration and MW of HA are reduced. Loss of normal HA disrupts the stability of the cartilage matrix, reduces the homeostatic and chondroprotective effects of HA, and reduces the viscoelasticity of the synovial fluid, increasing the susceptibility of the joint to damage by biomechanical forces. ${ }^{4,6,9}$ Low MW HA may also have a proinflammatory effect that accelerates progression of OA joint damage. ${ }^{10}$

Under normal conditions, throughout life, the molecular size, volume, and shape of HA in synovial fluid remain the same. However, with age, there is a sharp and progressive drop in the concentration of HA and in the elastic and viscous properties of synovial fluid such that, in older individuals, synovial fluid is nonelastic and viscous. Altered elastoviscosity despite maintenance of HA structure suggests that the interaction between neighboring molecules of HA changes with age.

In comparison, in synovial fluid aspirated from knee OA joints, the limiting viscosity number, which is an indicator of the size, volume, and shape of $1 \mathrm{HA}$ molecule and its interaction with its solvent fluid, as well as the MW of HA are significantly reduced. In patients with $\mathrm{OA}$, the viscous and elastic behaviors of synovial fluid are considerably below normal values. ${ }^{11}$

These observations provide the rationale behind intraarticular injection of HA for the management of OA. In theory, the aim is to improve joint lubrication and viscosity of the synovial fluid. However, recent studies indicate that the protective physiochemical functions of exogenous HA may confer disease-modifying, long-term effects on articular cartilage, associated with long-lasting symptom relief particularly in the initial phases of OA..$^{8,12,13}$

\section{Mechanism of action of exogenous HA supplementation}

Although there is limited evidence for symptomatic benefit of orally administered exogenous HA in knee OA, because of its MW, HA is poorly absorbed from the gastrointestinal tract (the oral bioavailability is around 5\%) and the usual route of administration is injection directly into the joint. ${ }^{13-15}$

Although the clinical benefits of intra-articular HA supplementation were initially thought to be consequent to direct augmentation of synovial fluid elasticity and viscosity, this is unlikely to be true as the intra-articular half-life of injected HA is short, being no longer than 48 hours for most viscosupplements. ${ }^{16,17}$ Of these, Hylan G-F 20 has the highest MW, which extends the joint residence time to at least 7 days, although it may still be detectable in the cartilage and synovial tissues for up to 28 days. ${ }^{18}$ In contrast, the clinical benefits of viscosupplementation in terms of pain and mobility are long lasting (clinical studies show effects lasting up to a year after a single injection), and improvement in HA concentration has been demonstrated for up to 6 months postinjection with significantly increased viscoelasticity. ${ }^{17,19,20}$

In vitro and in vivo studies have provided evidence for a number of biological mechanisms that might explain these discrepant observations, some of which may be dependent on the dose and MW of the exogenous HA (Table 1). In addition to its structural and mechanical roles, HA acts as a signaling molecule. It interacts with receptors on the surfaces of a range of different cells, including extracellular matrix fragments, chondrocytes, osteocytes, synoviocytes, and immune cells, regulating cell proliferation, migration, and differentiation. ${ }^{1,9,12,13}$ Effects of HA include promotion of endogenous HA production and enhanced synthesis of extracellular matrix proteins, including chondroitin and keratin sulfate, and PG. ${ }^{1,17}$ HA suppresses cartilage damage by reducing the activity of fibronectin fragments, which would normally bind and penetrate cartilage where they increase levels of MMP and inhibit PG synthesis. HA reduces the production and activity of various inflammatory mediators, including cytokines, proteases, and prostaglandins, and inhibits MMP activity such that cell matrix synthesis is favored over degradation. High MW HA has antioxidant effects that protect articular chondrocytes from damage by oxygen-derived free radicals. HA may reduce NO production in a tissue-specific fashion, reducing its inflammatory activity in the menisci and synovium.

In addition to its effects on inflammatory mediators, HA may also directly affect the function and behavior of immune cells, influencing cell proliferation, motility, aggregation, and adherence and phagocytosis.

Following intra-articular injection, high MW HA accumulates in cartilage and subchondral bone. ${ }^{13}$ Histological evidence shows that it prevents degradation of cartilage and may promote its regeneration. In patients with symptomatic $\mathrm{OA}$ of the knee, HA injection preserves knee cartilage, as measured by both cartilage volume and cartilage defects and by reduced markers of cartilage degradation in synovial fluid..$^{1,921}$ 
Table I Biological effects of HA

\begin{tabular}{|c|c|c|}
\hline Effects on extracellular matrix & Effects on immune cells & Effects on inflammatory mediators \\
\hline $\begin{array}{l}\text { - Enhanced HA synthesis } \\
\text { - Enhanced synthesis of PG and chondroitin } \\
\text { sulfate } \\
\text { - Suppressed PG release from extracellular } \\
\text { matrix } \\
\text { - Prevents PG breakdown from cartilage } \\
\text { - Reduced markers of cartilage breakdown } \\
\text { (chondroitin 4- and 6-sulfates) }\end{array}$ & $\begin{array}{l}\text { - Inhibits proliferation and activation } \\
\text { - Reduced motility of lymphocytes and } \\
\text { macrophages } \\
\text { - Inhibits phagocytosis } \\
\text { - Suppresses aggregation of neutrophils } \\
\text { - Inhibits adhesion and neutrophil-associated } \\
\text { cartilage destruction }\end{array}$ & $\begin{array}{l}\text { - Reduced levels of } \\
\text { ○ Prostaglandins } \\
\text { ○ Leukotrienes } \\
\text { - Increased production of cAMP } \\
\text { - Reduced expression/activity of } \\
\text { ○ IL-I, IL-6 } \\
\text { ○ Stromelysin } \\
\text { ○ TNF- } \alpha \\
\text { ○ TIMP-I (inhibits MMP) } \\
\text { - Plasminogen activator } \\
\text { - Reduced arachidonic acid release } \\
\text { - Antioxidant effects } \\
\text { - Reduced production of NO }\end{array}$ \\
\hline
\end{tabular}

Note: Data from Moreland,' Abate and Salini, ${ }^{6}$ and Kusayama et al. ${ }^{9}$

Abbreviations: HA, hyaluronic acid; PG, proteoglycans; cAMP, cyclic adenosine monophosphate; IL-I, interleukin-I; IL-6, interleukin-6; TNF- $\alpha$, tumor necrosis factor $\alpha$; TIMP-I, tissue inhibitor of metalloproteinases-I; MMP, metalloproteinase; NO, nitric oxide.

MW appears to play an important role in determining the effects of intra-articular HA. Hylan G-F 20 has a high molecular weight (6-7 million $\mathrm{Da}$ ) and, in comparison to alternative lower MW HA preparations, retains higher amounts of fluid in the articular space. Anti-inflammatory activity, measured by inflammatory cell migration in the joint and reduced prostaglandin $\mathrm{E} 2$ and bradykinin concentration, was also greater with Hylan G-F $20{ }^{6}$

\section{HA effects on nociception}

In patients with knee OA, inflammation influences excitability of nociceptors of articular nerves, causing them to discharge spontaneously and become hyperalgesic and sensitive to nonnoxious joint movement. The intensity of acute pain experienced during noxious stimulation of peripheral tissues is proportional to the firing frequency of nociceptor fibers. In experimental models of OA, administration of high MW HA significantly decreased ongoing nerve activity and movement-evoked nociception in normal and inflamed joints. Hylan G-F 20 reduced impulse activity in sensory fibers evoked by movements within and outside the working range of both normal and inflamed joints by $\sim 70 \%$. In contrast, HA solutions with lower MW and hence lower viscoelasticity were less or ineffective. ${ }^{22}$ It is speculated that highly viscoelastic HA reduces the sensitivity of stretch-activated ion channels involved in depolarization of the articular nerve terminal in response to mechanical forces. ${ }^{1,22}$ The long-term significance of this finding in human osteoarthritic joints is uncertain.

Anti-inflammatory effects of HA are likely to contribute to pain relief. In a prospective study of patients with OA of the knee, intra-articular injection of HA was associated with significant improvements in pain and function accompanied by age-dependent improvements in fluid viscosity at higher shear speeds and reduced inflammatory cytokines. Reduction in pain severity correlated with the change in IL- $1 \beta$ levels by month 6 . The investigators speculated that the effects of HA are most likely consequent to a combination of favorable improvements in pro- and anti-inflammatory cytokines, viscosity and oxidative damage in the synovial fluid..$^{23}$

Substance $\mathrm{P}$ is a tachykinin neuropeptide that plays a role in pain and joint inflammation. It is secreted by peripheral sensory neurons, nerves in the spinal cord, and inflammatory cells such as macrophages, eosinophils, lymphocytes, and dendritic cells. In comparison to joints with mild arthritis, those with severe arthritis have been shown to contain an increased density of substance-P-containing sensory neurons and higher substance $\mathrm{P}$ content. ${ }^{24}$ Through its interaction with excitatory amino acids, prostaglandins, and NO, HA may indirectly affect the pharmacology of substance $P$ and has been shown to inhibit increased vascular permeability induced by substance P. ${ }^{1}$

\section{Clinical effectiveness of viscosupplementation}

Two meta-analyses indicate that intra-articular HA is at least as effective or more effective than oral NSAIDs, COX-2 inhibitors, paracetamol or intra-articular corticosteroids in terms of improving pain and function. ${ }^{25,26}$ The most recent meta-analysis, including 137 studies comprising 33,243 participants, reported standardized mean differences for pain, function, and stiffness at 3-month follow-up. For pain, 
all interventions were better than placebo, with the most efficacious being intra-articular HA (effect size 0.63) and the least effective being paracetamol (effect size 0.18). ${ }^{26}$ All interventions except intra-articular corticosteroids were more effective than placebo in terms of functional improvement, and NSAIDs, COX-2 inhibitors, and intra-articular HA had a significantly greater effect on stiffness than placebo. ${ }^{26} \mathrm{An}$ effect size $>0.2$ is considered to be clinically relevant for individuals with chronic pain conditions such as OA of the knee. ${ }^{25}$

However, expert consensus is that because viscosupplements differ widely in terms of origin, MW, molecular structure, method of cross-linking, rheological behavior, and formulation, it may not necessarily be appropriate to extrapolate clinical results from one to another. Therefore, efficacy and safety for individual products needs to be assessed from randomized controlled trials. ${ }^{8}$

\section{Studies of Hylan G-F 20 in patients with OA of the knee}

A Cochrane review of viscosupplementation included 24 randomized controlled trials of Hylan G-F 20 published up until 2005. ${ }^{27}$ In general, Hylan G-F 20 was significantly more effective than placebo in terms of pain control with weight-bearing, at night, at rest, and with movement. Hylan G-F 20 was significantly more effective than NSAIDs for pain relief but was not significantly different for functional outcomes. Two studies in the Cochrane review concluded that Hylan G-F 20 plus appropriate care provided significantly better improvement in pain and function in comparison to appropriate care alone. The number needed to treat for patient global assessment, based on effectiveness rated as good or satisfactory, was $4 .{ }^{27}$

Additionally, another study in the Cochrane review showed a statistically significant beneficial effect of Hylan G-F 20 for pain on walking and for function with a longer duration of action over 26 weeks follow-up in comparison with intra-articular injection of triamcinolone hexacetonide. ${ }^{27}$ The number needed to treat for responders (from 5 to 13 weeks postinjection) was 5 . In contrast, there was no difference in pain or functional outcomes with Hylan G-F 20 in comparison to intra-articular injection of betamethasone sodium phosphate at 6 months. ${ }^{28}$ In general, intra-articular corticosteroid injections appear to be more effective for the first 4 weeks of treatment, with comparable efficacy from week 4-8, whereafter exogenous HA provides greater pain reduction. ${ }^{28,29}$ de Campos et $\mathrm{al}^{29}$ randomized 104 patients with knee OA to receive a single injection of Hylan G-F 20 either alone or in combination with intra-articular triamcinolone hexacetonide. At Week 1, symptom and function scores were significantly better in the combination group, but from Week 4-24 there were no differences between groups. There were no differences in adverse events between groups. Therefore, combining a corticosteroid with intra-articular Hylan G-F 20 may improve early symptom relief, but does not add to symptom relief in the longer term.

Data from comparative trials of Hylan G-F 20 with low MW HA are conflicting. Although some did not demonstrate differences in outcomes, others suggest that Hylan G-F 20 may be more effective and act more rapidly with longer lasting effects. ${ }^{27,28}$

A prospective randomized trial compared the clinical effectiveness, functional outcomes, and patient satisfaction of intra-articular injection of Hylan G-F 20 with sodium hyaluronate (MW 0.5-0.73 million Da) in 392 patients with OA of the knee. ${ }^{30}$ Hylan G-F 20 was administered as a series of 3 weekly injections and sodium hyaluronate as a series of 5 weekly injections. The majority of patients had grade III OA. In the Hylan G-F 20 group, knee pain measured using the visual analog scale (VAS) improved from 6.7 to 3.2 at 6 weeks $(P=0.02)$, and the improvement was sustained at 1 year (VAS score $3.7, P=0.04$ ). In the sodium hyaluronate group, pain improved from 6.6 baseline to 5.7 at 6 weeks ( $P$ not significant) and 4.1 at 3 months $(P=0.04)$, but improvement was not sustained at 6 months (VAS score 5.9). Change from baseline pain VAS scores was significantly different between groups at 6 weeks and 6 months, but not significantly different at 3 months. Similar results were observed for Western Ontario and McMaster Universities Osteoarthritis Index (WOMAC) pain subscales. There was an improvement in WOMAC physical activity subscale in both groups, which was significantly better in the Hylan G-F 20 group at 6 and 12 months. The WOMAC stiffness subscale was improved to a similar degree in both groups at 3,6 and 12 months. Overall, patients were generally more satisfied with their treatment in the Hylan G-F 20 group.

Another 1-year study suggested that Hylan G-F 20 was clinically effective and cost-effective. ${ }^{31}$ Ambulatory patients with radiologically verified OA of the knee and a VAS total pain score $>175$ of $500 \mathrm{~mm}$ on the WOMAC pain scale despite treatment with paracetamol or NSAIDs were allocated to guideline-directed appropriate care with $(n=127)$ or without $(n=128) 3$ intra-articular injections of Hylan $G-F$ 20 at 1 week intervals. Although intra-articular injections added to the overall cost of treatment, reduction in pain scores $(-38 \%$ vs $-13 \% ; P<0.0001)$ and the percentage of patients with improvement in overall WOMAC scores $(62 \%$ 
vs $35 \% ; P=0.0001)$ were significantly greater in patients receiving Hylan G-F 20 (Table 2). Hylan G-F 20 patients also required fewer intra-articular corticosteroid injections, less NSAID therapy, and fewer other medications for knee $\mathrm{OA}$, and therefore had fewer adverse events and less need for medication to treat gastrointestinal side effects. The Hylan G-F 20 group reported significantly better improvements in global evaluations measuring overall OA and overall health and in health-related quality of life instruments, which reflect the health status of the whole patient. Thus, the investigators conclude that increased costs of treatment associated with Hylan G-F 20 are offset by better health-related quality of life. $^{31,32}$

Pal et $\mathrm{al}^{20}$ reported results from an open-label 1-year study in which they evaluated the efficacy and safety of a single intra-articular injection of Hylan G-F 20 in 394 patients with grade I-III OA of the knee. Significant improvements in all efficacy parameters (pain, stiffness and physical function) were observed from as early as 1 week after treatment, with $>30 \%$ reduction in walking pain, the primary outcomes measure, at 52 weeks. At baseline, only $15 \%$ of patients rated their global OA condition as "well" or "very well," vs almost $60 \%$ at week 26 and $65 \%$ at week 52 . The corresponding percentages for physician's rating of patients' global condition were $16 \%, 60 \%$, and $64 \%$. At 26 and 52 weeks, $88 \%$ and $98 \%$ of patients, respectively, reported no change in use of concomitant medications. Local adverse events around the target knee (primarily arthralgia and synovitis) were reported by $6 \%$ of patients, but only half of these were considered treatment related.

A retrospective review of medical records suggests that viscosupplementation with Hylan G-F 20 can delay surgery in patients who are candidates for total knee replacement (TKR). ${ }^{33}$ Instead of being scheduled for immediate TKR, Hylan G-F 20 was administered to 1,187 knees (863 patients) with severe (grade IV) OA. The incidence and median time to TKR was 19\% and 638 days (minimum 14 days, maximum 2,147 days), respectively. Among the patients who had not yet undergone TKR, the median time of Hylan G-F 20 treatment was 810 days (minimum 7 days, maximum 2,222 days). Survival analysis indicated that $75 \%$ of knees had not required TKR by 3.8 years. Fifty-five percent of knees required only 1 course of injections, and 14\% required more than 2 courses. Age was the only covariate that was significantly associated with time to TKR, with the highest incidence and shortest time to TKR in the age group 60-69 years. The injections were well tolerated, with local adverse events occurring in $1.2 \%$. Twelve percent $(228 / 1,342)$ of patients experienced synovitis or knee pain.

The majority of cases of synovitis were mild (36.8\%) to moderate (43\%), with $10 \%$ being severe. There were no serious adverse events, ie, requiring hospitalization, knee surgery, permanent limb dysfunction, or death. The study has multiple limitations including its retrospective nature, selection bias, single center nature, and lack of placebo-controlled arm.

Altman et $\mathrm{al}^{34}$ investigated the effect of Hylan G-F 20 on time to TKR surgery in patients with OA. This study was a retrospective analysis of medical records of 182,022 patients with knee OA. Of these patients with knee OA, 50,349 $(27.7 \%)$ were users of HA, receiving $\geq 1$ courses of HA prior to TKR, 131,673 (72.3\%) were non-HA users. The mean time to TKR for the patients not receiving $\mathrm{HA}$, patients receiving 1 course of $H A$, and patients receiving $\geq 5$ courses of HA was $0.7,1.4$, and 3.6 years, respectively. Although promising with respect to HA delaying time to TKR, this study is limited by

Table 2 Outcomes at I year (percentage of patients) for appropriate care with and without Hylan G-F 20 in knee OA

\begin{tabular}{|c|c|c|c|c|}
\hline Outcomes at I year & $\begin{array}{l}\text { Appropriate care } \\
\text { plus Hylan G-F } 20 \\
(n=127)\end{array}$ & $\begin{array}{l}\text { Appropriate } \\
\text { care alone } \\
(n=128)\end{array}$ & $\begin{array}{l}\text { Relative } \\
\text { benefit increase } \\
(95 \% \mathrm{CI})\end{array}$ & $\begin{array}{l}\text { NNT } \\
(95 \% \mathrm{CI})\end{array}$ \\
\hline Improved WOMAC pain & $69 \%$ & $40 \%$ & $72 \%(36-121)$ & $3(3-6)$ \\
\hline Improved WOMAC pain and stiffness or physical functioning & $62 \%$ & $35 \%$ & $77 \%(36-134)$ & $4(3-7)$ \\
\hline Improved OA in study knee & $73 \%$ & $27 \%$ & $168 \%(10 \mid-265)$ & $2(2-3)$ \\
\hline Improved $\mathrm{OA}$ in all joints & $38 \%$ & $17 \%$ & $120 \%(43-243)$ & $5(3-10)$ \\
\hline Absence of side effects & $62 \%$ & $41 \%$ & $53 \%(20-98)$ & $5(3-11)$ \\
\hline \multicolumn{5}{|l|}{ 'Patients reporting medication use: } \\
\hline Intra-articular corticosteroid injections in study knee & $14 \%$ & $70 \%{ }^{\mathrm{a}}$ & & \\
\hline Intra-articular corticosteroid injections in other knee & $6 \%$ & $27 \%^{\mathrm{a}}$ & & \\
\hline Oral NSAIDS & $65 \%$ & $79 \%$ & & \\
\hline
\end{tabular}

Notes: ${ }^{a} P<0.000$ I; ${ }^{b} P=0.0062$. ${ }^{c}$ Part data from Raynauld et al. ${ }^{31}$ Partially adapted with permission from Symmons $D$. Hylan G-F 20 was safe and effective in knee osteoarthritis and had a relatively low cost-utility ratio. ACP J Club. 2003; I38(I):20.32

Abbreviations: OA, osteoarthritis; NNT, number needed to treat; WOMAC, Western Ontario and McMaster Universities Osteoarthritis Index; NSAIDs, nonsteroidal anti-inflammatory drugs; $\mathrm{Cl}$, confidence interval. 
its retrospective nature and use of records which cannot be verified or standardized between treating physicians and their evaluations and reasons for decision for TKR. In addition, the reasons for patients receiving/not receiving HA may have influenced the outcomes.

Boutefnouchet et $\mathrm{al}^{35}$ studied the effect of Hylan G-F 20 on need for arthroplasty or other surgical interventions for OA of the knee. Seventy-seven consecutive patients were followed up for 5 years. ${ }^{35}$ After 1 year, 71 (87\%) of patients were considered as responders. At 5-year follow-up, 41 patients $(50 \%)$ were classified as responders. There were no reported adverse events during the study period. The authors concluded that Hylan G-F 20 may help delay need for arthroplasty in those patients not ready for arthroplasty. The study was limited by its retrospective nature and was not placebo controlled. Furthermore, the patients included in the study had a mix of tibiofemoral compartment disease. Some patients had maintained joint space while others had tibiofemoral compartment disease with bone-on-bone changes and were unfit or too young for arthroplasty. Thus, the delay in need for arthroplasty may have been influenced by patients being too young or medically unfit. Furthermore, the lack of adverse events in this study may reflect inadequate reporting as compared to total absence of adverse events.

Given the limitations of the above studies, it is difficult to quantify benefit of viscosupplements to delay time to knee replacement surgery. There is a need for a robust prospective, sham-controlled, double-blind study.

\section{International guidelines on positioning of viscosupplementation in therapy of knee OA}

There are a large number of national and international guidelines and consensus statements on the management of knee $\mathrm{OA}$. There remains a nonalignment between guidelines with respect to the recommended role of viscosupplementation for knee OA.

The consensus statement on the European Society for Clinical and Economic Aspects of Osteoporosis and Osteoarthritis algorithm for the management of knee OA suggests that HA be positioned after NSAIDs, and considers these agents safe and effective. ${ }^{36}$ In contrast, the National Institute of Health and Care Excellence (NICE) specifically recommends against the use of intra-articular viscosupplementation. ${ }^{37}$

The Osteoarthritis Research Society International guidelines for the nonsurgical management of knee OA provide an "uncertain" recommendation based on the heterogeneity of study results in the literature. ${ }^{38}$
The Royal Australian College of General Practitioners states that there is evidence to suggest that hyaluronic acid is of some benefit for OA of the knee. ${ }^{39}$

The American College of Rheumatology (ACR) initially conditionally recommended the use of hyaluronate injections for $\mathrm{OA}$ of the knee in patients with inadequate response to initial therapy. ${ }^{40}$ However, in a subsequently published position statement, ACR recommended the use of intra-articular hyaluronic acid injection for the treatment of OA of the knee in adults, and stated that hyaluronic acid injection is clinically indicated for management of $\mathrm{OA}$ in patients who are not good candidates for surgery or who do not respond to other treatment options. ${ }^{41}$

In contrast, a task force of European experts on OA recommend that viscosupplementation should not be reserved for patients in whom NSAIDs have failed or are contraindicated, but may be considered for early OA because of potential chondoprotective effects, and may be used in advanced cases of OA where surgical intervention is too risky or where the patient declines surgical intervention. ${ }^{8}$

\section{Conclusion}

Clinical studies indicate that viscosupplementation is an effective local treatment for OA, with a high rate of responders among those with $\mathrm{OA}$ of the knee. It improves both pain and function, while sparing concomitant use of anti-inflammatory treatment and corticosteroids. Patients usually tolerate the injection well and, with careful administration and use of appropriate aseptic technique, the rate of complications is low.

There remains a need for robust scientific studies to help reduce the uncertainty around viscosupplementation and its role in a management strategy for OA. At the current time, the place of viscosupplementation in therapy and the decision as to which patient may be a candidate remains an individualized choice for discussion between the patient and treating physician.

\section{Author contribution}

All authors contributed toward information analysis, drafting and critically revising the paper and agree to be accountable for all aspects of the work.

\section{Disclosure}

Dr Poobalan Naidoo is employed as a medical advisor for Sanofi. Sanofi manufactures and markets Hylan G-F 20 (Synvisc ${ }^{\circledR}$ ). Dr David Webb is a medical writer and has received payment for services to numerous pharmaceutical 
companies, including Sanofi. The authors report no other conflicts of interest in this work.

\section{References}

1. Moreland LW. Intra-articular hyaluronan (hyaluronic acid) and hylans for the treatment of osteoarthritis: mechanisms of action. Arthritis Res Ther. 2003;5(2):54-67.

2. Ma VY, Chan L, Carruthers KJ. Incidence, prevalence, costs, and impact on disability of common conditions requiring rehabilitation in the United States: stroke, spinal cord injury, traumatic brain injury, multiple sclerosis, osteoarthritis, rheumatoid arthritis, limb loss, and back pain. Arch Phys Med Rehabil. 2014;95(5):986.e1-995.e1.

3. Woolf AD, Pfleger B. Burden of major musculoskeletal conditions. Bull World Health Organ. 2003;81(9):646-656.

4. Goldberg VM, Goldberg L. Intra-articular hyaluronans: the treatment of knee pain in osteoarthritis. J Pain Res. 2010;3:51-56.

5. Funck-Brentano T, Cohen-Solal M. Subchondral bone and osteoarthritis. Curr Opin Rheumatol. 2015;27(4):420-426.

6. Abate M, Salini V. Hyaluronic acid in the treatment of osteoarthritis: what is new? In: Chen Q, editor. Osteoarthritis - Diagnosis, Treatment and Surgery. Rijeka: InTech; 2012:102-122.

7. Snibbe JC, Gambardella RA. Use of injections for osteoarthritis in joints and sports activity. Clin Sports Med. 2005;24(1):83-91.

8. Henrotin Y, Raman R, Richette P, et al. Consensus statement on viscosupplementation with hyaluronic acid for the management of osteoarthritis. Semin Arthritis Rheum. 2015;45(2):140-149.

9. Kusayama Y, Akamatsu Y, Kumagai K, Kobayashi H, Aratake M, Saito T. Changes in synovial fluid biomarkers and clinical efficacy of intra-articular injections of hyaluronic acid for patients with knee osteoarthritis. J Exp Orthop. 2014;1:16.

10. Roque V, Agre M, Barroso J, Brito I. Managing knee ostheoarthritis: efficacy of hyaluronic acid injections. Acta Reumatol Port. 2013;38(3):154-161.

11. Balazs EA. The physical properties of synovial fluid and the special role of hyaluronic acid. In: Helfet A, editor. Disorders of the Knee. 2nd ed. Philadelphia: JB Lippincott; 1974:63-75.

12. Necas J, Bartosikova L, Brauner P, Kolar J. Hyaluronic acid (hyaluronan): a review. Veterinární Medicína. 2008;53(8):397-411.

13. du Souich P. Absorption, distribution and mechanism of action of SYSADOAS. Pharmacol Ther. 2014;142(3):362-374.

14. Moller I, Martinez-Puig D, Chetrit C. LB012 oral administration of a natural extract rich in hyaluronic acid for the treatment of knee OA with synovitis: a retrospective cohort study. Clin Nutr Suppl. 2009;4(2):171-172.

15. Kalman DS, Heimer M, Valdeon A, Schwartz H, Sheldon E. Effect of a natural extract of chicken combs with a high content of hyaluronic acid (Hyal-Joint $\left.{ }^{\circledR}\right)$ on pain relief and quality of life in subjects with knee osteoarthritis: a pilot randomized double-blind placebo-controlled trial. Nutr J. 2008;7(1):3.

16. Brown TJ, Laurent UB, Fraser JR. Turnover of hyaluronan in synovial joints: elimination of labelled hyaluronan from the knee joint of the rabbit. Exp Physiol. 1991;76(1):125-134.

17. Bagga H, Burkhardt D, Sambrook P, March L. Longterm effects of intraarticular hyaluronan on synovial fluid in osteoarthritis of the knee. J Rheumatol. 2006;33(5):946-950.

18. Stitik TP, Kazi A, Kim JH. Synvisc $®$ in knee osteoarthritis. Future Rheumatol. 2008;3(3):215-222.

19. Balazs EA, Denlinger JL. Viscosupplementation: a new concept in the treatment of osteoarthritis. J Rheumatol Suppl. 1993;39(Suppl 39):3-9.

20. Pal S, Thuppal S, Reddy KJ, et al. Long-term (1-year) safety and efficacy of a single 6-mL injection of Hylan G-F 20 in Indian patients with symptomatic knee osteoarthritis. Open Rheumatol J. 2014;8:54-68.

21. Wang Y, Hall S, Hanna F, et al. Effects of Hylan G-F 20 supplementation on cartilage preservation detected by magnetic resonance imaging in osteoarthritis of the knee: a 2-year single-blind clinical trial. $B M C$ Musculoskelet Disord. 2011;12:195.
22. Gomis A, Pawlak M, Balazs EA, Schmidt RF, Belmonte C. Effects of different molecular weight elastoviscous hyaluronan solutions on articular nociceptive afferents. Arthritis Rheum. 2004;50(1):314-326.

23. Vincent HK, Percival SS, Conrad BP, Seay AN, Montero C, Vincent KR. Hyaluronic acid (HA) viscosupplementation on synovial fluid inflammation in knee osteoarthritis: a pilot study. Open Orthop J. 2013;7:378-384.

24. O'Connor TM, O'Connell J, O’Brien DI, Goode T, Bredin CP, Shanahan $\mathrm{F}$. The role of substance $\mathrm{P}$ in inflammatory disease. J Cell Physiol. 2004;201(2):167-180.

25. Bannuru RR, Natov NS, Dasi UR, Schmid CH, Mcalindon TE. Therapeutic trajectory following intra-articular hyaluronic acid injection in knee osteoarthritis - meta-analysis. Osteoarthritis Cartilage. 2011;19(6):611-619.

26. Bannuru RR, Schmid CH, Kent DM, Vaysbrot EE, Wong JB, McAlindon TE. Comparative effectiveness of pharmacologic interventions for knee osteoarthritis: a systematic review and network meta-analysis. Ann Intern Med. 2015;162(1):46-54.

27. Bellamy N, Campbell J, Welch V, Gee TL, Bourne R, Wells GA. Viscosupplementation for the treatment of osteoarthritis of the knee. Cochrane Database Syst Rev. 2006;2:CD005321.

28. Migliore A, Giovannangeli F, Granata M, Laganà B. Hylan G-F 20: review of its safety and efficacy in the management of joint pain in osteoarthritis. Clin Med Insights Arthritis Musculoskelet Disord. 2010;3:55-68.

29. de Campos GC, Rezende MU, Pailo AF, Frucchi R, Camargo OP, Pires O. Adding triamcinolone improves viscosupplementation: a randomized clinical trial. Clin Orthop Relat Res. 2013;471(2):613-620.

30. Raman R, Dutta A, Day N, Sharma HK, Shaw CJ, Johnson GV. Efficacy of Hylan G-F 20 and sodium hyaluronate in the treatment of osteoarthritis of the knee - a prospective randomized clinical trial. Knee. 2008;15(4):318-324.

31. Raynauld JP, Torrance GW, Band PA, et al. A prospective, randomized, pragmatic, health outcomes trial evaluating the incorporation of hylan G-F 20 into the treatment paradigm for patients with knee osteoarthritis (part 1 of 2): clinical results. Osteoarthritis Cartilage. 2002;10(7):506-517.

32. Symmons D. Hylan G-F 20 was safe and effective in knee osteoarthritis and had a relatively low cost-utility ratio. ACP J Club. 2003;138(1):20.

33. Waddell DD, Bricker DC. Total knee replacement delayed with Hylan G-F 20 use in patients with grade IV osteoarthritis. J Manag Care Pharm. 2007;13(2):113-121.

34. Altman R, Lim S, Steen RG, Dasa V. Hyaluronic acid injections are associated with delay of total knee replacement surgery in patients with knee osteoarthritis: evidence from a large US Health Claims Database. PLoS One. 2015;10(12):e0145776.

35. Boutefnouchet T, Puranik G, Holmes E, Bell KM. Hylan GF-20 viscosupplementation in the treatment of symptomatic osteoarthritis of the knee: clinical effect survivorship at 5 years. Knee Surg Relat Res. 2017;29(2):129-136.

36. Bruyère $\mathrm{O}$, Cooper $\mathrm{C}$, Pelletier JP, et al. A consensus statement on the European Society for Clinical and Economic Aspects of Osteoporosis and Osteoarthritis (ESCEO) algorithm for the management of knee osteoarthritis-From evidence-based medicine to the real-life setting. Semin Arthritis Rheum. 2016;45(4 Suppl):S3-S11.

37. National Institute for Health and Clinical Excellence. NICE Guideline on Osteoarthritis: The Care and Management of Osteoarthritis in Adults, NICE Clinical Guideline 177. London: National Institute for Health and Clinical Excellence; 2014. Available from: http://guidance.nice.org.uk/ CG177. Accessed November 30, 2017.

38. McAlindon TE, Bannuru RR, Sullivan MC, et al. OARSI guidelines for the non-surgical management of knee osteoarthritis. Osteoarthritis Cartilage. 2014;22(3):363-388.

39. The Royal Australian College of General Practitioners Working Group. Guideline for the Non-Surgical Management of Hip and Knee Osteoarthritis. Melbourne: The Royal Australian College of General Practitioners; 2009. Available from: https://www.racgp.org.au/yourpractice/guidelines/musculoskeletal/hipandkneeosteoarthritis. Accessed December 1, 2017. 
40. Hochberg MC, Altman RD, April KT, et al. American College of Rheumatology 2012 recommendations for the use of nonpharmacologic and pharmacologic therapies in osteoarthritis of the hand, hip, and knee. Arthritis Care Res. 2012;64(4):465-474.
41. American College of Rheumatology. Intra-Articular Hyaluronic Acid Injection in Osteoarthritis of the Knee. Atlanta, GA: American College of Rheumatology; 2012. Available from: https://www.rheumatology.org/ Portals/0/Files/Viscosupplementation.pdf. Accessed December 1, 2017.
Orthopedic Research and Reviews is an international, peer-reviewed, open access journal that focusing on the patho-physiology of the musculoskeletal system, trauma, surgery and other corrective interventions to restore mobility and function. Advances in new technologies, materials, techniques and pharmacological agents are particularly welcome. The manuscript management system is completely online and includes a very quick and fair peer-review system, which is all easy to use. Visit http://www.dovepress.com/testimonials.php to read real quotes from published authors. 